Instituto Internacional de Investigación y Desarrollo Tecnológico Educativo INDTEC, C.A.

DOI: https://doi.org/10.29394/scientific.issn.2542-2987.2017.2.4.6.106-123

OAl-PMH: http://www.indteca.com/ojs/index.php/Revista Scientific/oai

\title{
Huerto Familiar como Estrategia Ambiental para Promover el Desarrollo Sustentable con Representantes
}

\author{
Autor: Eliseo Ramón Castillo Camacho \\ Universidad Pedagógica Experimental Libertador, UPEL \\ eliseo1974castillo@hotmail.com \\ Barinas, Venezuela
}

\section{Resumen}

La presente investigación tuvo como propósito promover el huerto familiar como estrategia ambiental en el desarrollo sustentable con padres y representantes de la Unidad Educativa "Nerio Torres Alvarado" del sector el Pueblito, parroquia Barinitas, municipio Bolívar del estado Barinas. La naturaleza del estudio estuvo enmarcada bajo el enfoque cualitativo en una Investigación Acción Participativa. Por otro lado, la investigación se realizó en (4) cuatro fases: Fase I. Preparatoria (Reflexión y Diseño), Fase II. El trabajo de campo, Fase III. Analítica, Fase IV. Informativa. Los informantes claves fueron: (03) representantes. En la investigación se utilizó como técnica la observación participante, la entrevista a profundidad y como instrumento una guía de entrevista y cuaderno de campo. Desde el punto de vista del método de investigación acción participativa, siendo esta una forma específica de interacción social que tiene por objeto recolectar datos para la indagación, permitiendo el contacto y la recolección de opiniones, ideas y preocupaciones de los padres y representantes. Finalmente, por todo lo expuesto, la presente investigación constituye un aporte significativo al proceso de enseñanza y aprendizaje, ya que es competencia de la familia y de los docentes como agentes promotores de difusión de conocimientos en dicho proceso.

\footnotetext{
Palabras clave: huerto familiar; estrategia ambiental; desarrollo
sustentable. Palabras clave: huerto familiar; estrategia ambiental; desarrollo
sustentable.
} 


\title{
Familiar Vegetable Garden as an Environmental Strategy to Promote the Sustainable Development in Parents and Representatives
}

\begin{abstract}
This research aimed to promote the family garden as an environmental strategy in sustainable development with parents and representatives of the Educational Unit "Nerio Torres Alvarado" of the sector El Pueblito, Barinitas parish, Bolivar municipality of Barinas state. The nature of the study was framed under the qualitative approach in a Participatory Action Research. On the other hand, the research was done in (4) four phases: Phase I. Preparatory (Reflection and Design), Phase II. Fieldwork, Phase III. Analytical, Phase IV. Informative. Key informants were: (03) representatives. The research used as a technique participant observation, the interview in depth and as an instrument an interview guide and field notebook. From the point of view of the participatory action research method, this is a specific form of social interaction that aims to collect data for the inquiry, allowing the contact and collection of opinions, ideas and concerns of parents and representatives. Finally, for all of the above, the present research constitutes a significant contribution to the teaching and learning process, since it is the competence of the family and the teachers as promoters of dissemination of knowledge in this process.
\end{abstract}

Keywords: family garden; environmental strategy; sustainable development. 


\section{Introducción}

El huerto familiar se tiene como uno de los factores primordiales en el ámbito de desarrollo familiar, económico de productividad agraria, constituye una alternativa de producción sustentable, ya que son espacios que no acceden más de los $500 \mathrm{~m}^{2}$, lo cual permite un manejo adecuado del agro ecosistema, dependiendo de los espacios de terrenos útiles para la actividad de siembra y que se conocen por su clasificación en sus diferentes densidades y tipos, generalmente se constituyen en los aledaños o patios de los hogares, que permite la producción de todo tipo de plantas ya sean medicinales, comestibles, ornamentales, condiméntales, frutales, maderables, otras. Por tal motivo, constituyen la posibilidad de que las familias se favorezcan con la obtención de rubros necesarios para complementar la cesta alimentaria, producidos por ellos mismos con rubros más fresco, sanos y de calidad.

Asimismo, el huerto familiar contribuye con la posibilidad de tener empleo a partir de la comercialización de dichos rubros, actualmente las familias en su mayoría obtienen productos provenientes de otros lugares a mayor costo, situación que perjudica desde punto de vista económico y de salud, en nuestro caso algunas veces es causa de deserción escolar. Es por ello, que se pretende tomar el huerto familiar como referencia estratégica en la búsqueda de solución al problema enmarcado en la siguiente investigación, donde se busca formar a padres y representantes para la promoción del desarrollo sustentable, y que además sus hogares se transformen en una aula de clase por y con la enseñanza de sus representados en la implementación del huerto familiar y la actividad agraria como una alternativa de sustento familiar y cultural, manteniéndose así de generación en generación dicha actividad, por su puesto en armonía con nuestro ambiente.

Esta investigación, se desarrollará bajo la modalidad de investigación de campo, enmarcada dentro de una investigación descriptiva de tipo cualitativa, debido a que el contexto del artículo, es una situación realista, tal 
como lo afirma Kelinger (citado por Hernández, Fernández y Batista (2003), al decir que es "un estudio de investigación en una situación realista que poseen mayor validez externa que los experimentos de laboratorio" (pág. 72).

Tomando en cuenta lo antes expuesto, permitió brindar la orientación adecuada ya que la investigación se basó en las realidades de los padres y representantes para la resolución del problema planteado, como es el escaso desarrollo sustentable en el sector el "Pueblito", municipio Bolívar del estado Barinas. Con el fin de fortalecer o aportar algunos conocimientos objetivos y confiables para contribuir en la consolidación del desarrollo sustentable, como base fundamental en el fortalecimiento de la soberanía alimentaria en el núcleo familiar y comunitario.

Esto contribuye en la formación de los padres y representantes, y a su vez va a influir de manera positiva en el aprendizaje de sus hijos, por lo que la formación puede ser entendida según Soto (1.999), como:

Un conjunto de actividades; derecho de todo sujeto; dinámica de desarrollo personal; ponerse en forma; adquisición de conocimientos personales; proceso unificador de la evolución humana; proceso activo que requiere la mediación de otros; proceso de transformación; proceso social de desarrollo personal; un proceso eminentemente de adquisición de conocimientos personales, entre otros (pág. 137).

En tal sentido, se consideraron las políticas emanadas por el Ministerio del Poder Popular para la Educación, con la intención de formar el nuevo ciudadano fortaleciendo el sentido de pertenencia por el ambiente, con el buen uso de los recursos naturales en la práctica del trabajo de campo-agrícola, o en cualquier proyecto de vida que pueda permitir el desarrollo familiar y social.

Por tal motivo, se tiene previsto tomando en cuenta la formación de los padres y representes en materia agrarialimentaria desde la escuela a sus hogares involucrando lineamientos educativos, como es la inclusión de los todos los actores sociales en diferentes actividades en la Unidad Educativa 
Nerio Torres Alvarado, a tal efecto se formulan las interrogantes de investigación: ¿Cuáles son los conocimientos que tienen los padres y representantes sobre el huerto familiar como estrategia de gestión ambiental para el desarrollo sustentable? ¿Cómo diseñar la implementación del huerto familiar como estrategia de gestión ambiental para el desarrollo sustentable con los padres y representantes de la Unidad Educativa Nerio Torres Alvarado, sector el Pueblito, municipio Bolívar del estado Barinas? ¿Cómo ejecutar la implementación del huerto familiar como estrategia de gestión ambiental para el desarrollo sustentable con los padres y representantes de la Unidad Educativa Nerio Torres Alvarado, sector el Pueblito, municipio Bolívar del estado Barinas? ¿Cuáles serían los avances en el desarrollo sustentable a partir de la implementación huerto familiar?

\subsection{Propósito General}

Promover el huerto familiar como estrategia de gestión ambiental para el desarrollo sustentable con los padres y representantes de la Unidad Educativa "Nerio Torres Alvarado", sector el Pueblito, municipio Bolívar del estado Barinas.

\section{Teoría y Conceptos}

\subsection{Antecedentes de la Investigación}

En este caso como el término lo indica, se refiere al conocimiento brindado por trabajos de investigación realizados por otros autores, según Arias, (2006) señala que:

se refiere a todos los trabajos de investigación que anteceden al nuestro, es decir, aquellos trabajos donde se hayan manejado las mismas categorías o propuestos objetivos similares; además sirven de guía para el investigador y le permiten hacer comparaciones y tener ideas sobre cómo se trató el problema en esa oportunidad (pág. 32). 
De esta manera la presente investigación busca fortalecer la temática en estudio para orientar el proceso asegurando que dicha investigación se base en un contenido seguro y confiable en la formación y promoción de la realización de huertos familiares para el desarrollo sustentable con los padres y representantes de la Unidad Educativa Nerio Torres Alvarado. El presente artículo se apoya en algunas investigaciones previas recogidas en diversos estudios realizados sobre el tema en cuestión.

Moreno, (2011), en su trabajo de grado que se denomina; Incidencia de la Cooperación Internacional en las estrategias de Desarrollo Sustentable (CIDES) en Venezuela.

En lo metodológico y dada la carencia de estudios similares, esta investigación favorece los conocimientos resultantes de un análisis documental del marco conceptual disponible y la construcción de partes teóricas inexistentes y necesarias. Es por ello, que se da a conocer el objetivo general, basado en realizar un análisis crítico de la actuación que ha tenido la participación de dos grandes actores de la cooperación internacional en la implementación del nuevo paradigma del desarrollo en Venezuela, para el periodo 1994 al 2010. Así mismo favorece la identificación de experiencias y buenas prácticas producto de esfuerzos conjugados a nivel internacional (macro), nacional (meso) y local (micro) y del estudio del cuadro normativo del desarrollo sustentable vinculándolos con los de la cooperación internacional.

Así mismo, Cárdenas, (2011), en su trabajo de investigación denominado "Estrategias Pedagógicas y de Sensibilización en la Educación Ambiental"; en la Escuela Técnica Agropecuaria El Algarrobo, del Estado Barinas.

Los objetivos que orientaron dicha investigación son: (a) fomentar estrategias pedagógicas y de sensibilización para la Educación Ambiental en la Escuela Técnica Agropecuaria El Algarrobo, (b) comprender la situación educativa ambiental en esta institución, (c) propiciar manejos ambientales en 
el desarrollo curricular de la asignatura Educación Ambiental hacia el desarrollo sustentable, como resultado de las experiencias vividas con los actores claves, (d) interpretar el grado de sensibilización de los actores comprometidos en el proceso para la resolución de los problemas ambientales.

De esta manera, se orientó en la siguiente metodología basándose en el estudio cualitativo, a través del método Investigación-Acción. Se apoyó en el Paradigma Crítico Reflexivo y la manera de abordarlo es en espiral y continuo, de acuerdo con Kemmis y Mc Taggart posee un diseño estructurado en cuatro (4) fases: observación, planificación, acción y reflexión. En esta investigación la entrevista constituye el instrumento principal de recolección de información, manteniendo el formato de preguntas abiertas y personales. Los sujetos del estudio son un grupo de 52 participantes.

Igualmente, Calderón (2013), en su investigación denominada, "Conocimiento Tradicional sobre Plantas Medicinales en Huertos Familiares en la Mixteca Poblana, México", plantea como analizar el papel que juegan las plantas medicinales en los huertos familiares, y los conocimientos alrededor de ellas. Con este estudio pretende contribuir al diseño de estrategias de desarrollo rural que promuevan alternativas para el desarrollo local de las comunidades

Por tal motivo la metodología se basa en el trabajo de campo realizado desde el 2010 al 2013 en dos (2) diferentes entornos, uno rural y otro urbano, en el municipio de Tepexi de Rodríguez, Puebla, México. (Agua de la Luna, Huejonapan, y Tula) y en el sur de la Ciudad de Puebla, México. Se generaron tres (3) artículos de investigación. El primero trata sobre el conocimiento tradicional que tienen los niños de plantas medicinales y los procesos de transmisión; el siguiente sobre las plantas medicinales cultivadas en los huertos familiares y el último sobre las decisiones de salud y los motivos de las personas para utilizar plantas medicinales o acudir con el médico.

Por otra parte, Reyes (2014). En su trabajo de investigación 
denominado: Agrobiodiversidad y Manejo del Huerto Familiar, su Contribución a la Seguridad Alimentaria, en una Localidad del Municipio de Paso de Ovejas, Veracruz.

De qué nos alimentamos, y cómo accedemos a los alimentos, es una de nuestras principales preocupaciones como individuos y sociedad, las cifras siguen siendo significativamente altas, 870 millones de personas (12.7\%) a nivel mundial, se encuentran desnutridos. En México el crecimiento de la población y las inadecuadas políticas gubernamentales, han traído como consecuencia el incremento de comunidades vulnerables que ingresan al grupo de pobreza, y por consecuencia, a la dificultad para acceder a alimentos y dietas adecuadas. El objetivo del presente artículo, fue analizar la agrobiodiversidad y manejo del huerto familiar y su contribución a la seguridad alimentaria. Éste se sustentó en una revisión bibliográfica y en un modelo de gestión, que comprende las etapas de: sensibilización, diagnosis, y desarrollo comunitario, realizadas bajo la conjunción de los enfoques cualitativo y cuantitativo, a través de distintas metodologías tales como entrevistas semiestructuradas, observaciones participantes realizadas en campo y talleres de intercambio de saberes. Esto permitió conocer la diversidad y funcionalidad que poseen los huertos familiares, los alimentos que conforman al patrón alimentario de la comunidad Bandera de Juárez, así como el porcentaje de alimentos provenientes del huerto familiar en la dieta diaria de las familias de la comunidad.

\subsection{Huertos Familiares}

Es necesario e importante resaltar, las conceptualizaciones de aspectos o variables, de un trabajo de investigación para enfocar las orientaciones acertadas del mismo según Reyes (2014) señala que:

Los Huertos Familiares son agroecosistemas tradicionales con un arraigo importante en las culturas indígenas y campesinas, 
tanto de regiones templadas como tropicales, configuran una de las primeras formas agrícolas, estos han evolucionado a través de los años, y se representan como una opción cultural y material profundamente arraigada al territorio. Que siguen vigentes en la actualidad y se encuentran mejor representados en las áreas tropicales (pág. 17).

Por lo antes señalado, el huerto familiar represento una estrategia fundamental dentro de la investigación por cuanto ofreció una alternativa socio-productiva y cultural dentro de la formación de los padres y representantes en la búsqueda del desarrollo sustentable como eje fundamental en la presente investigación

\subsection{Ambiente}

Así mismo el ambiente, como elemento fundamental en el desarrollo integral de los seres humanos y demás organismos vivos, manifiesta la importancia de ser tomado en cuenta en todo proyecto de vida, tal es el caso de la presente investigación según, la ley orgánica del ambiente, (2006) en su artículo 3 ambiente:

Conjunto o sistema de elementos de naturaleza física, química, biológica o socio cultural, en constante dinámica por la acción humana o natural que rige y condiciona la existencia de los seres humanos y demás organismos vivos, que interactúan permanentemente en un espacio y tiempo determinado (pág. 2).

En tal sentido, la presente investigación tiene como uno de sus objetivos principales dentro de la formación de los actores sociales, promover el sentido de pertenencia ambiental con el uso adecuado de los recursos naturales en la elaboración de los huertos familiares, contribuyendo así a la reciprocidad en el interés común de retribuir todo lo que la naturaleza nos brinda. 


\subsection{Desarrollo sustentable}

De la misma manera, en la conceptualización del desarrollo sustentable se podría decir que es la conjugación de la praxis bien ejecutada de las anteriores variables (huerto familiar y ambiente), el cual fue acertado en la presente investigación según, la Comisión Mundial para el Medio Ambiente y el Desarrollo (1983) establecida por las Naciones Unidas, definió el desarrollo sustentable como el "desarrollo que satisface las necesidades del presente sin comprometer las capacidades que tienen las futuras generaciones" ( $s / p)$.

Por consecuencia, en la ejecución y logros alcanzados en todas las actividades planificadas, se pudo evidenciar el equilibrio acertado entre hombre- natura constituyéndose así la formación de los padres y representantes en la búsqueda del desarrollo sustentable, como interés superior en la presente investigación.

\section{Recorrido Metodológico}

En este espacio es donde se da a conocer la metodología que permitió desarrollar el presente artículo, definiendo las acciones destinadas a describir el problema en estudio, tomando como referencia el planteamiento que según Hurtado (2012) manifiesta que:

Se entiende por metodología el estudio de los modos o maneras de llevar a cabo una actividad determinada. Por tanto, incluye el estudio de los métodos técnicas, tácticas, estrategias y los procedimientos que utiliza el investigador para lograr los objetivos del trabajo (pág. 141).

Ante estos señalamientos, el autor orienta al investigador a precisar las maneras y modos de ir ejecutando todo lo que versa durante el proceso de estudio, con el objetivo de organizar la estructura adecuada que constituya un contenido objetivo y confiable de la investigación, con la buena acertación y 
utilización de los distintos elementos y herramientas metodológicas que conllevaron la veracidad y concreción del problema planteado.

A tal efecto, la presente investigación se desarrolló bajo la modalidad de investigación de campo, descriptiva de tipo cualitativa, bajo el enfoque de la investigación acción participativa como lo indica Ander, (2003) establece que: "el método de la investigación acción participativa (IAP) combina dos procesos, el de conocer y el de actuar, implicando en ambos a la población cuya realidad se aborda" (pág. 35).

Por tal motivo, la población sujeto de estudio durante el proceso de investigación, fueron padres y representantes de la Unidad Educativa Nerio Torres Alvarado, tomando en cuenta sus realidades, culturas y costumbres en la búsqueda del desarrollo sustentable. De igual manera, se mencionan que las técnicas aplicadas se basaron en la observación participante, entrevista a profundidad e instrumento: guía de preguntas y cuaderno de campo, que permitieron recabar la información necesaria para el desarrollo de las diferentes líneas estratégicas de la presente investigación.

Con respecto, al análisis de los resultados según Gonzales y Cano (2010), lo refieren como:

el proceso a través del cual vamos más allá de los datos para acceder a la esencia del fenómeno de estudio, es decir, a su entendimiento y comprensión; el proceso por medio del cual el investigador expande los datos más allá de la narración descriptiva (pág. 132).

En este sentido, dichos resultados se basaron en lo aportado por los informantes claves durante el proceso de investigación, por medio de las técnicas antes mencionadas, las cuales permitieron emerger aspectos importantes relegando las tareas a la categorización, codificación y teorización, por medio de la unidad de análisis, que contribuyo ala respectiva sistematización acertada. Por consiguiente, la fiabilidad y confirmabilidad en 
términos de investigación cualitativas se realizó por medio de la triangulación desde las perspectivas de los representantes y dos profesionales en materia agrícola del Instituto Nacional de capacitación y educación (INCE).

\section{Conclusiones y Recomendaciones}

\subsection{Conclusiones}

En este sentido, la investigación se inclinó en la búsqueda de la promoción del desarrollo sustentable el cual fue desarrollada por los padres y representantes de la unidad educativa Nerio Torres Alvarado, quienes fueron formados no solo en la elaboración de los huertos familiares como unidad productiva en el hogar sino que también, se formaron en la comprensión de la importancia del ambiente como eje principal en la estructura de la creación de cualquier proyecto de vida, cabe destacar que los padres y representantes en las diferentes interacciones teóricas y prácticas durante la ejecución del artículo, mostraron mucho interés a medida que fueron conociendo la diversidad de contenido específico concerniente a los temas, pues para ellos (padres y representantes), era satisfactorio saber que podrían ser los próximos docentes de sus hijos al impartir esos conocimientos en sus hogares, para tal fin se hace mención de los logros alcanzados desarrollados en cuatro objetivos de la siguiente manera.

1.- En lo que concierne al diagnóstico, se pudo constatar que algunos padres y representantes tenían conocimiento sobre lo que era un huerto escolar, mientras que otros no, también se denoto la variedad de espacios con que contaban los aledaños (patios) de sus hogares que permitieron la actividad agrícola como elemento primordial para la realización del huerto, además los padres y representantes dieron a conocer la necesidad de implementar alguna alternativa que pudiese apalear los gastos del hogar. Situación por la cual los padres y representantes lograron constituir, la alternativa antes manifestada con la implementación de los huertos familiares aprovechando los espacios en 
sus hogares útiles para la siembra, tomando en cuenta el uso racional de los recursos naturales y conociendo la importancia del ambiente.

2.- En el ámbito del diseño de las acciones a realizar, se lograron establecer con los padres y representantes las actividades a desarrollar durante el proceso de investigación permitiendo esto la participación directa de los sujetos en estudio, de esta manera se tomó en cuenta el tiempo de la colectividad participante debido a que algunos de los representantes tienen trabajo fijo, los mismos mostraron mucho interés por la unión y compromiso manifiesto durante el desarrollo de las reuniones realizadas, en tal sentido se mostró uno de los aspectos importantes de la investigación como es la integración familiar y de la sociedad, ya que los huertos familiares fungen como sistema de producción, permitiendo el intercambio de la misma.

3.- En la continuación del desarrollo de la investigación y la aplicación de las acciones, los padres y representantes conocieron de manera práctica y teórica los diferentes temas a desarrollar como fueron: los huertos familiares, educación ambiental, desarrollo sustentable; que esto conllevo a una formación integral, que no era solo el utilizar los huertos familiares como unidad de producción sino, conocer el equilibrio adecuado en la utilización de los recursos naturales y el respeto con el ambiente, de esta manera se logró que los sujetos en estudios conocieran que cuando se habla de desarrollo sustentable engloba una serie de aspectos, que conllevan a la reciprocidad universal donde el beneficio no se debe enmarcar con el interés de uno sino del colectivo, orientando el camino de que se mantenga en el tiempo para el beneficio continuo de generaciones venideras.

4.- En cuanto al seguimiento y control, que conllevo a la evaluación continua de las acciones planificadas, los padres y representantes además de mostrar el conocimiento obtenido, también pudieron conseguir alcanzar las metas establecidas con la elaboración de los huertos familiares y sus beneficios, como es la producción de algunos rubros sembrados durante el 
proceso del desarrollo de las actividades planificadas, además de la organización comunitaria en cuanto a las labores culturales (desmalezar, riego, aplicación de abono orgánico entre otros), actividades propias de los huertos dentro del cuido de la siembra, para tal fin los padres y representantes se organizaron en lo que ellos llamaron "comité de huertos" cuya función predomina además del apoyo continuo de rutina, pautaron ofrecer el cuido pertinente a los demás huertos en caso de ausencia en los demás hogares por cualquier circunstancia (vacaciones entre otros motivos), que pudiera perjudicar la siembra en su momento; esto mostro la integración familiar y el compromiso de los padres y representantes, como indicador organizacional en la búsqueda del desarrollo sustentable, desprendido del aprendizaje integral enmarcado en la presente investigación.

\subsection{Recomendaciones}

En consecuencia, una vez conocida las conclusiones se darán a conocer algunas recomendaciones, según la Universidad Pedagógica Experimental Libertador (U.P.E.L), (2010) en las recomendaciones que el autor fórmula deben contribuir a la solución del problema o al mejoramiento cualitativo de la práctica en sus procesos o resultados (pág. 38).

En consecuencia, las recomendaciones van dirigidas a la extensión de la solución de la problemática antes planteada, puesto que, al incrementarse la utilización del huerto familiar como estrategia para promocionar el desarrollo sustentable, permite una mejor situación económica y por ende mejor calidad de vida, que se establecen de la siguiente manera.

1.- Implementar los huertos familiares en los hogares, pertenecientes al radio de acción de las demás instituciones educativas, como alternativa de sustentabilidad familiar.

2.- Se debe realizar jornadas de formación de manera periódica para incentivar a los padres y representantes, a participar en las labores agrícolas. 
3.- Los huertos familiares, pueden ser utilizados como una alternativa de motivación a los niños y niñas que tengan vocación por el sector agrícola, para que de esta manera a corto, mediano o largo plazo se formen a nivel profesional y pueda contarse con personal calificado que permita extender esa formación a la sociedad; cabe destacar que es de vital importancia fortalecer la implementación de producción agrícola en nuestro país.

4.- Los padres y representantes, una vez que cuenten con la formación correspondiente pueden transformarse en el docente desde sus hogares, promocionando esos conocimientos dentro del proceso de enseñanzaaprendizaje de sus propios hijos, puesto que los huertos familiares son el espacio de unión familiar y de formación de valores ambientalistas y culturales, que enmarca el sentido de pertenencia, equilibrio en el uso de los recursos naturales para alcanzar el desarrollo sustentable como eje fundamental, en el marco de la superación del individuo.

\section{Referencias}

Arias, F. (2006). Técnicas e Instrumentos de Recolección de Información Guía para su Elaboración. 3ra. Ed. Caracas, Venezuela: Editorial. Episteme.

Ander, E. (2003). Repensando la Investigación-Acción Participativa (I.A.P). Cuarta Edición. Gobierno Vasco, España. Recuperado de: http://aulavirtual.agro.unlp.edu.ar/pluginfile.php/34553/mod resource/c ontent/1/10ANDER-EGG-Ezequiel-La-investigacion-propiamentedicha.pdf

Cárdenas, G. (2011). En su trabajo de investigación denominado: Estrategias Pedagógicas y de Sensibilización en la Educación Ambiental. Requisito parcial para optar al grado de Magister Scientiarum de la Universidad Nacional Experimental Ezequiel Zamora. 
Calderón, H. (2013). Trabajo de investigación denominado: Conocimiento Tradicional sobre Plantas Medicinales en Huertos Familiares. Tesis presentada como requisito parcial para obtener el grado de doctora en ciencias especialista en estrategias para el desarrollo agrícola regional presentado en la Poblana, México: Colegió de postgrado.

Gonzales, T., y Cano, A. (2010). Introducción al Análisis de Datos en Investigación Cualitativa: Tipos de Análisis y Proceso de Codificación (II). Revista Científica: Nure Investigación, N 44, ISSN: 1697-218X. Recuperado de:

http://www.nureinvestigacion.es/OJS/index.php/nure/article/view/476

Hernández, C. (2011). El modelo de la contabilidad ecológica y el desarrollo sostenible. Recuperado de:

http://www.gestiopolis.com/modelo-de-la-contabilidad-ecologica-y-eldesarrollo-sostenible/

Hurtado, J. (2012). Metodología de la Investigación. Cuarta edición. Editorial Quirón. Bogotá-Caracas.

Ley orgánica del ambiente (2006). Gaceta Oficial № (5833E) del 22 de diciembre. [Documento en línea]. Recuperado de:

http://www.iclam.gov.ve/pdf/leyes amb/13 ley org amb.pdf

Moreno, J. (2011). Incidencia de la Cooperación Internacional en las Estrategias de Desarrollo Sustentable en Venezuela. Trabajo de Investigación que se Presenta para Optar al Grado de Doctor en Estudio de Desarrollo. Caracas, Venezuela.

Reyes, A. (2014). En su trabajo de investigación denominado: Agrobiodiversidad y Manejo del Huerto Familiar, su Contribución a la Seguridad Alimentaria, en una Localidad del Municipio de Paso de Ovejas, Veracruz. Tesis: para obtener el grado de maestra en ecología tropical. Universidad Veracruzana. 
Soto, M. (1999). Grupos de formación. En grupos y Dispositivos de Formación. Buenos Aires, Argentina: Ediciones Novedades Educativas. Universidad de Buenos Aires.

Universidad Pedagógica Experimental Libertador (2010). Manual de Trabajos de Grado de Especialización y Maestría y Tesis Doctorales. (4ta. ed.). Caracas: FEDUPEL. 


\section{Eliseo Ramón Castillo Camacho}

e-mail: eliseo1974castillo@hotmail.com

Fecha de Nacimiento 11 de noviembre de 1974, natural

de Barinas, Venezuela, soltero. Estudios: Primaria:

Escuela Básica Bolivariana Barinitas. Secundaria: 7mo y 8vo Escuela Básica Bolivariana Barinitas. Secundaria:

3ero a 5to año Liceo Nocturno Cándido Antonio Meza.

Pregrado: LCDO. En Educación: Mención Integral en la

Universidad Nacional Experimental de los Llanos (UNELLEZ), Barinas. Postgrado: Cursante Maestría: Educación Ambiente y Desarrollo, UPEL, Barinas. Desempeño: Docente de Aula: Unidad Educativa Primaria Nerio Torres Alvarado, Barinitas, 2008-2013. Docente: Apoyo Técnico: Defensoría educativa, Barinitas, 2013-2016, ubicada en Barinitas, Municipio Bolívar Estado Barinas.

El contenido de este manuscrito se difunde bajo una Licencia de Creative Commons ReconocimientoNoComercial-Compartirlgual 4.0 Internacional 